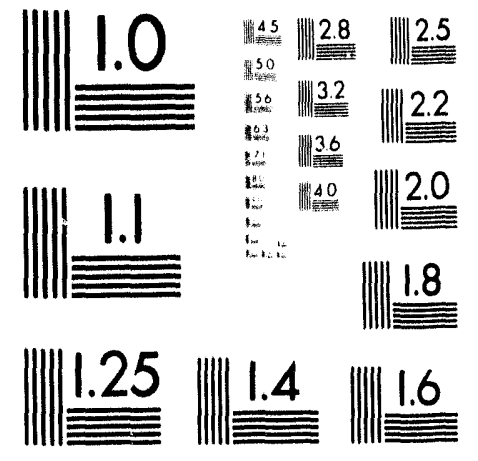


The submutred manuscript has been outhored by a contractor of the U.S. Government under contrucl No. DE.

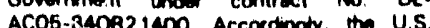
ACOS-340A2 1400. Accordinglv. Ho U.S. Government relens Accing nomaxcherve. rovaity-tree heonice to publish or reproduce the publuthed form of this contribution, of allow others to do so. for U.S. Government purposes:"

\section{STABILITY OF AN UNCOOLED SEGMENT OF A HIGH-TEMPERATURE SUPERCONDUCTOR*}

\author{
Lawrence Dresner \\ Oak Ridge National Laboratory \\ P.O. Box 2009, Oak Ridge, TN 37831-8040
}

\section{ABSTRACT}

If the level of a liquid cryogen inadvertently falls below the top of a magnet winding, it may expose a segment of the conductor. Depending on the length of the uncooled segment and on how the matrix resistivity varies with temperature, zero, one, or two steady normal states may be possible. The stability of the various steady states, the conditions under which they appear, their energies of formation, and the voltages they produce are studied in this paper.

\section{INTRODUCTION}

When a magnet is cooled by a liquid cryogen (e.g., $\mathrm{LHe}$ or $\mathrm{LN}_{2}$ ), the liquid level may inadvertently fall below the top of the winding and expose a segment of the conductor. If the uncooled segment becomes normal, it may not be able to recover the superconducting state owing to its lack of cooling, but its temperature may also not rise indefinitely because its ends are clamped at the saturation temperature of the liquid cryogen. The ultimate fate of such uncooled segments is the subject of this paper.

The composite ceramic superconductors operate in a range of temperatures in which their physical properties (specific heat, matrix resistivity, maurix thermal conductivity) vary strongly with temperature. This strong variation complicates the analysis, but it cannot be ignored: the conventional assumption of constant properties often used in the analysis of the low-temperature superconductors is inadmissible here.

\section{STEADY STATES}

The steady states of an uncooled segment are given by the solutions of the timeindependent heat balance equation

$$
\frac{d}{d z}\left(k \frac{d T}{d z}\right)+\rho J^{2} g(T)=0,
$$

where $g(T)$ is the current-sharing function that gives the fraction of the current flowing in the matrix. The particular solutions that interest us are even ones that obey the boundary conditions

"Research sponsored by the Office of Advanced Utility Concepts, Superconducting Technology Program, U.S. Department of Energy, under contract DE-AC05-840R21400 with Martin Marietta Energy Systems, Inc.

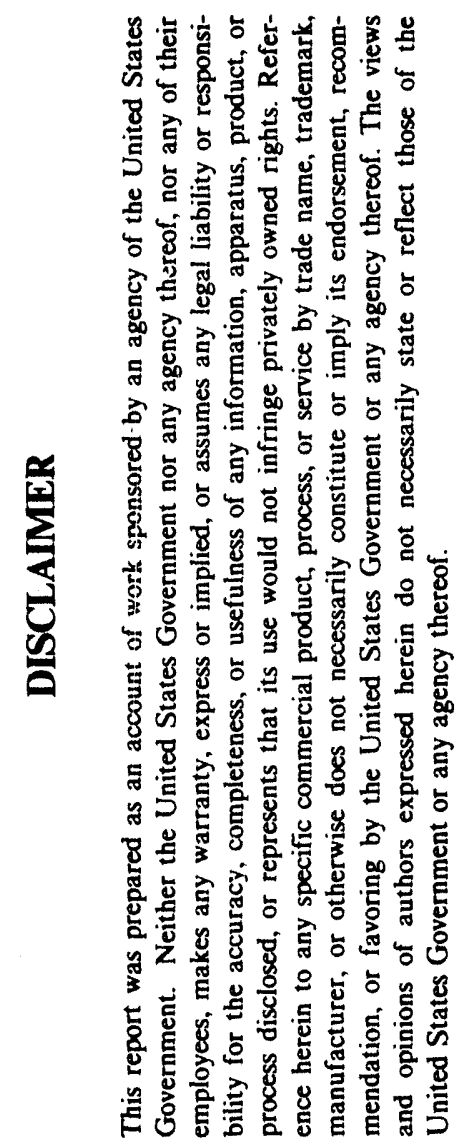




$$
T( \pm a)=T_{\mathrm{b}},
$$

where $2 a$ is the length of the uncooled segment. To simplify the explicit determination of the solutions, we shall ignore current sharing and assume that the conductor is completely normal over the entire uncooled length, recovering the superconducting state instantaneously as it plunges back into the liquid cryogen. Furthermore, we shall assume the matrix conductivity $k$ and resistivity $\rho$ are connected by the Wiedemann-Franz law

$$
k \rho=L_{0} T \quad\left(L_{0}=2.45 \times 10^{-8} \mathrm{~V}^{2} \mathrm{~K}^{-2}\right) .
$$

Then if we multiply Eq. (1a) by $k$, replace $g(T)$ by 1 , and set $q=k(d T / d z)$, we obtain

$$
q \frac{d q}{d T}+L_{0} J^{2} T=0
$$

[N.B.: $\left.k \frac{d}{d z}\left(k \frac{d T}{d z}\right)=k \frac{d T}{d z} \frac{d}{d T}\left(k \frac{d T}{d z}\right)=q \frac{d q}{d T}\right]$. Integrating Eq. (3), we find

$$
q^{2}=L_{0} J^{2}\left(T_{\mathrm{m}}^{2}-T^{2}\right)
$$

where $T_{\mathrm{m}}$ is the maximum temperature occurring at $z=0$. Here we have used the requirement of symmetry that $q(0)=0$. Now replacing $q$ by its definition in terms of $T$, we have

$$
L_{0}^{1 / 2} J d z=-\frac{k d T}{\left(T_{\mathrm{m}}^{2}-T^{2}\right)^{1 / 2}}=-\frac{L_{0} T d T}{\left(T_{\mathrm{m}}^{2}-T^{2}\right)^{1 / 2} \rho}
$$

which, upon integration over $z$ from 0 to $a$, yields

$$
L_{0}^{-1 / 2} J a=\int_{T_{\mathrm{b}}}^{T_{\mathrm{m}}} \frac{T d T}{\left(T_{\mathrm{m}}^{2}-T^{2}\right)^{1 / 2} \rho} .
$$

\section{EVALUATION OF THE FUNCTIONAL DEPENDENCE OF $T_{\mathrm{m}}$ ON $a$}

As we shall see below, the functional dependence of $T_{\mathrm{m}}$ on $a$ is not entirely simple: for values of $a$ greater than a certain limiting value $a_{*}$ there may not be a solution for $T_{\mathrm{m}}$ (meaning no normal steady states can exist), while for smaller values of $a$ more than one value of $T_{\mathrm{m}}$ may satisfy Eq. (6) (meaning that there is more than one normal steady state). To simplify the evaluation of the right-hand side of Eq. (6) we introduce the auxiliary variables $y$ and $\theta$ defined by

$$
\begin{gathered}
y=T_{\mathrm{m}} / T_{\mathrm{b}} \\
T=T_{\mathrm{m}} \cos \theta .
\end{gathered}
$$

Then Eq. (6) becomes

$$
\frac{J a}{L_{0}^{1 / 2} T_{\mathrm{b}}}=y \int_{0}^{\arccos (y / y)} \frac{\cos \theta d \theta}{\rho}
$$


Now, over limited ranges of temperature, $\rho$ can be taken to vary with a power $n$ of $T$. The highest power that occurs in the Bloch-Grineisen theory is 5 ; the lowest is 1 . At very low temperatures, where the residual resistivity dominates, $n=0$. When $\rho$ varies as power of $T$, i.e., when

$$
\rho=\rho_{b}\left(T / T_{b}\right)^{n}=\rho_{b} y^{n} \cos ^{n} \theta
$$

Equation (8) becomes

$$
\frac{J a \rho_{\mathrm{b}}}{L_{0}^{1 / 2} T_{\mathrm{b}}}=\frac{1}{y^{n-1}} \int_{0}^{\arccos (1 / y)} \frac{d \theta}{\cos ^{n-1} \theta}
$$

The right-hand side of Eq. (9b) can be evaluated easily when $n=0,1,2,3,4$, and 5 . The respective results are

$$
\begin{array}{rlrl}
J a \rho_{\mathrm{b}} / L_{0}^{1 / 2} T_{\mathrm{b}} & =\sqrt{y^{2}-1} & (n=0) \\
& =\arccos (1 / y) & (n=1) \\
& =\frac{1}{y} \ln \left(y+\sqrt{y^{2}-1}\right) & (n=2) \\
& =\sqrt{y^{2}-1} / y^{2} & (n=3) \\
& =\frac{1}{2 y^{2}}\left[\sqrt{y^{2}-1}+\frac{1}{y} \ln \left(y+\sqrt{y^{2}-1}\right)\right](n=4) \\
& =\frac{y^{2}+2}{3 y^{4}} \sqrt{y^{2}-1} & (n=5)
\end{array}
$$

The results for $n=1,2,3,4$, and 5 are plotted in Fig. 1 with $J a \rho_{b} / L_{0}^{1 / 2} T_{b}$ as abscissa and $y=T_{\mathrm{m}} / T_{\mathrm{b}}$ as ordinate. Two things are immediately evident from these numerical results: (1) for $n=1,2,3,4$, and 5 , solutions for $y=T_{m} / T_{b}$ are possible only when $J a \rho_{b} / L_{0}^{1 / 2} T_{b}$ is less than some limiting value, and (2) for $n=2,3,4$, and 5 , two solutions for $y=T_{\mathrm{m}} / T_{\mathrm{b}}$ are possible. For any $n \geq 1$, (including non-integer values) there is a limiting value of $J a \rho_{b} / L_{0}^{1 / 2} T_{b}$; for any $n>1$, there are two solutions for $y=T_{m} / T_{b}$, while for $n=1$ there is one.

\section{DISCUSSION}

If the value of $\operatorname{Jap}_{\mathrm{b}} / L_{0}^{1 / 2} T_{\mathrm{b}}$ exceeds the limiting value, there is no steady state, and the temperature of the uncooled segment continues to rise indefinitely. In this case, if the presence of the normal segment is not detected, it will eventually destroy itself.

Figure 2 shows the limiting values of $\operatorname{Ja\rho }_{b} / L_{0}^{1 / 2} T_{b}$ for $1 \leq n \leq 5$. (When $n<1$, there is no limiting value, and a steady state always exists.) Let us evaluate the limiting length for a ceramic-silver superconductor cooled by liquid nitrogen. In the temperature range 


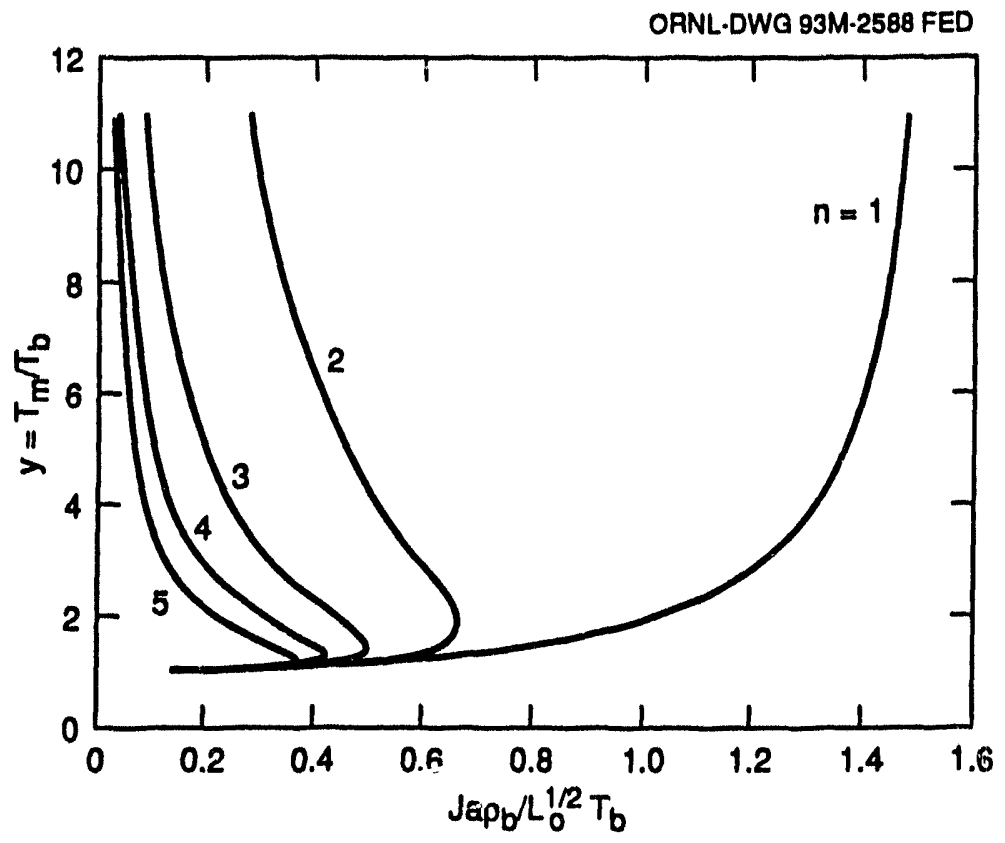

Figure 1. The dimensionless peak temperature $y=T_{\mathrm{m}} / T_{\mathrm{b}}$ plotted against the dimensionless uncooled length $J_{a p_{b}} / L_{0}^{1 / 2} T_{b}$ for $n=1(1) 5$.

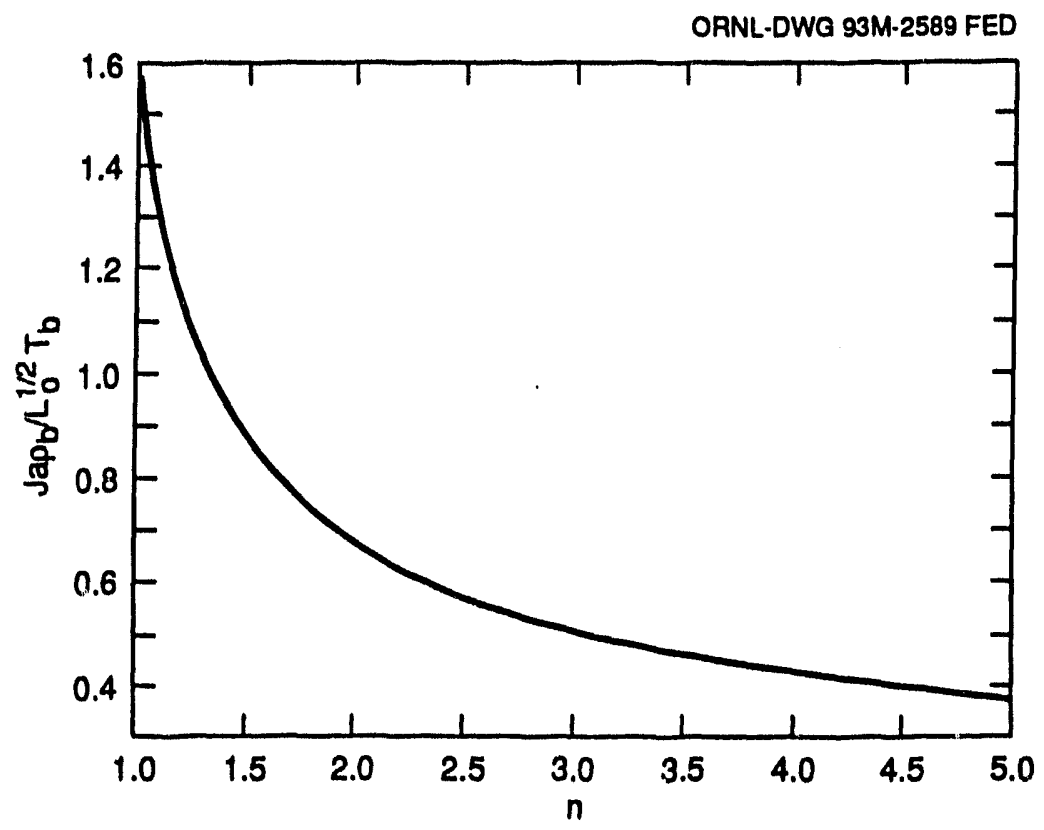

Figure 2. The limiting value of the dimensionless uncooled length $J a \rho_{b} / L_{0}^{1 / 2} T_{b}$ as a function of $n$ for $1 \leq n \leq 5$. 
$77 \mathrm{~K}-200 \mathrm{~K}$, the resistivity of silver varies closely as the $3 / 2$-power of the temperature, so we take $n=1.5$. The limiting value of $J a \rho_{b} / L_{0}^{1 / 2} T_{b}$ is then 0.845 . The resistivity $\rho_{b}$ of silver at $T_{\mathrm{b}}=77 \mathrm{~K}$ is $2.9 \times 10^{-9} \mathrm{ohm} \cdot \mathrm{m}$. If we take the current density in the silver in the normal state to be $5 \times 10^{7} \mathrm{~A} / \mathrm{m}^{2}$, we find $a=0.070 \mathrm{~m}=7.0 \mathrm{~cm}$, a comparatively short distance. In a recent $E P R I$ review, ${ }^{1}$ Moore has suggested that for high-field applications such as motors, generators, and energy storage, current densities an order of magnitude greater than that given above may be needed. In that case the limiting uncooled half-length may be less than $1 \mathrm{~cm}$.

The energy of formation $E$ of a steady state may be taken as a figure of merit that quantifies the ease with which such a state may be formed. Now

$$
E=2 \int_{0}^{a} d z \int_{T_{\mathrm{b}}}^{T} S\left(T^{\prime}\right) d T^{\prime}
$$

where $S$ is the volumetric heat capacity of the conductor. Now, just as we took the resistivity to vary as a power, $n$, of $T$ over a limited range of temperatures, so can we take the heat capacity to vary as a power, $m$, of $T$ over the same limited range. We now take

$$
\rho=\rho_{b}\left(T / T_{b}\right)^{n}, S=S_{b}\left(T / T_{b}\right)^{m},
$$

use Eq. (5) to change the variable of integration from $z$ to $T$, and finally introduce the quantities $y$ and $\theta$ defined in Eqs. (7); we find

$$
\frac{J E \rho_{b}}{L_{0}^{1 / 2} S_{b} T_{b}^{2}}=\frac{2}{m+1} \cdot \frac{1}{y^{n-1}} \int_{0}^{\arccos (1 / y)} \frac{(y \cos \theta)^{m+1}-1}{\cos ^{n-1} \theta} d \theta
$$

Now the heat capacity of both silver and BSCCO vary closely as the 0.5-power of $T$ in the temperature range $77 \mathrm{~K}-200 \mathrm{~K}$, so we take $m=0.5$ to continue our numerical example. The value of $y$ corresponding to the limiting value of $J a \rho_{b} / L_{0}^{1 / 2} T_{b}$ is 2.57 (so that $T_{\mathrm{m}}=198 \mathrm{~K}$ ). Then the right-hand side of Eq. (13) equals 2.03. The volumetric heat capacity of BSCCO is close to that of silver, ${ }^{2}$ and in the estimates being made here it is not worthwhile to distinguish them. For silver at $77 \mathrm{~K}, S_{\mathrm{b}}=1.69 \mathrm{MJ} / \mathrm{m}^{3}$. When $J=5 \times$ $10^{7} \mathrm{~A} / \mathrm{m}^{2}, E=22.0 \mathrm{MJ} / \mathrm{m}^{2}=22.0 \mathrm{~J} / \mathrm{mm}^{2}$. Thus the formation energy of the limiting steady state in a conductor with a $1-\mathrm{mm}^{2}$ cross section is $22.0 \mathrm{~J}$, which is quite substantial. It is therefore unlikely that a normal steady state would be created even if a $14-\mathrm{cm}$ segment of the conductor were uncooled.

The voltage, $V$, across such a normal steady state is given by

$$
V=2 \int_{0}^{a} \rho J d z=2 L_{0}^{1 / 2}\left(T_{\mathrm{m}}^{2}-T_{\mathrm{b}}^{2}\right)^{1 / 2}
$$

the second equality following from the same series of substitutions as given above. When $T_{\mathrm{m}}=198 \mathrm{~K}$ and $T_{\mathrm{b}}=77 \mathrm{~K}, V=57.1 \mathrm{mV}$, which though small, should be detectable. (N.B.: The voltage $V$ appears to be independent of $J$ and $\rho$, but in fact it is not: these quantities enter the determination of $T_{\mathrm{m}}$.)

So far, the discussion has been confined to the limiting length, for which only one steady state exists. For shorter lengths, two states can exist, and we need to determine whether both occur in practice. The upper state has the property that the peak temperature decreases as the uncooled length increases, so we are inclined-correctly as it turns out-to dismiss it. It can be shown that the upper steady state is unstable against small perturbations, which is why it cannot occur in practice: if it were ever created, the random 
thermal fluctuations always present would immediately destroy it. The lower state, in contrast, is stable against small perturbations and so can occur in practice. (The proofs of these assertions about stability are long and, owing to the page restriction on contributed papers, cannot be included here.)

Perhaps the most significant aspect of these calculations is the revelation that if $n>1$, no steady state may exist if the uncooled segment is too long. In that event, the conductor suffers a thermal runaway. A lower bound to the time over which this runaway occurs can be estimated from the adiabatic hot-spot formula

$$
J^{2} t=\int_{T_{b}}^{T_{\mathrm{m}}} \frac{S}{\rho} d T .
$$

The right-hand side of Eq. (15) has been calculated for Ag/BSCCO conductors in reference 2 . With a residual resistance ratio of $2500, T_{\mathrm{m}}=200 \mathrm{~K}$, and $T_{\mathrm{b}}=77 \mathrm{~K}$, it is roughly $4.0 \times 10^{16} \mathrm{~A}^{2} \mathrm{~m}^{-4} \mathrm{~s}$. When $J=5 \times 10^{7} \mathrm{~J} / \mathrm{m}^{2}, t=16 \mathrm{~s}$, this being a lower limit to the time available for detection and remedial action before the peak temperature reaches $200 \mathrm{~K}$. In the applications discussed by Moore in his review, the current densities were higher. When $J=5 \times 10^{8} \mathrm{~A} / \mathrm{m}^{2}$, for example, $t$ is only $1.6 \mathrm{~s}$. As may be recalled, in the example we have been following, the limiting length for this high current density is only $7.0 \mathrm{~mm}$. A cryogen vapor bubble trapped in a tight winding could easily create an uncooled segment this long. The saving grace in this case is the still rather large formation energy of the limiting steady state, namely, $2.2 \mathrm{~J} / \mathrm{mm}^{2}$.

What will happen in practice is still uncertain and awaits further construction of nitrogen-cooled magnets wound with high-temperature superconductors. But in any case, the simple formulas presented in this paper may aid in understanding behavior of such magnets.

\section{REFERENCES}

1. T. Moore, Superconductors are still hot, EPRI Journal 17:4-15 (September 1992).

2. L. Dresner, Stability and protection for $\mathrm{Ag} / \mathrm{BSCCO}$ magnets operated in the $20-40 \mathrm{~K}$ range, accepted for publication in Cryogenics.

\section{SYMBOLS}

$\begin{array}{ll}E & \text { Formation energy of a steady state } \\ J & \text { Current density } \\ L_{0} & \text { Lorenz constant } \\ S & \text { Volumetric heat capacity } \\ T & \text { Temperature } \\ T_{\mathrm{b}} & \text { Bath temperature } \\ T_{\mathrm{m}} & \text { Maximum temperature in uncooled segment } \\ V & \text { Voltage across an uncooled segment in steady state } \\ a & \text { Half-length of the uncooled segment } \\ g(T) & \text { Current sharing function } \\ k & \text { Thermal conductivity } \\ m & \text { Exponent in the power law of heat capacity versus temperature } \\ n & \text { Exponent in the power law of resistivity versus temperature } \\ q & k(d T / d z) \\ y & T_{\mathrm{m}} / T_{\mathrm{b}} \\ p & \text { Resistivity }\end{array}$



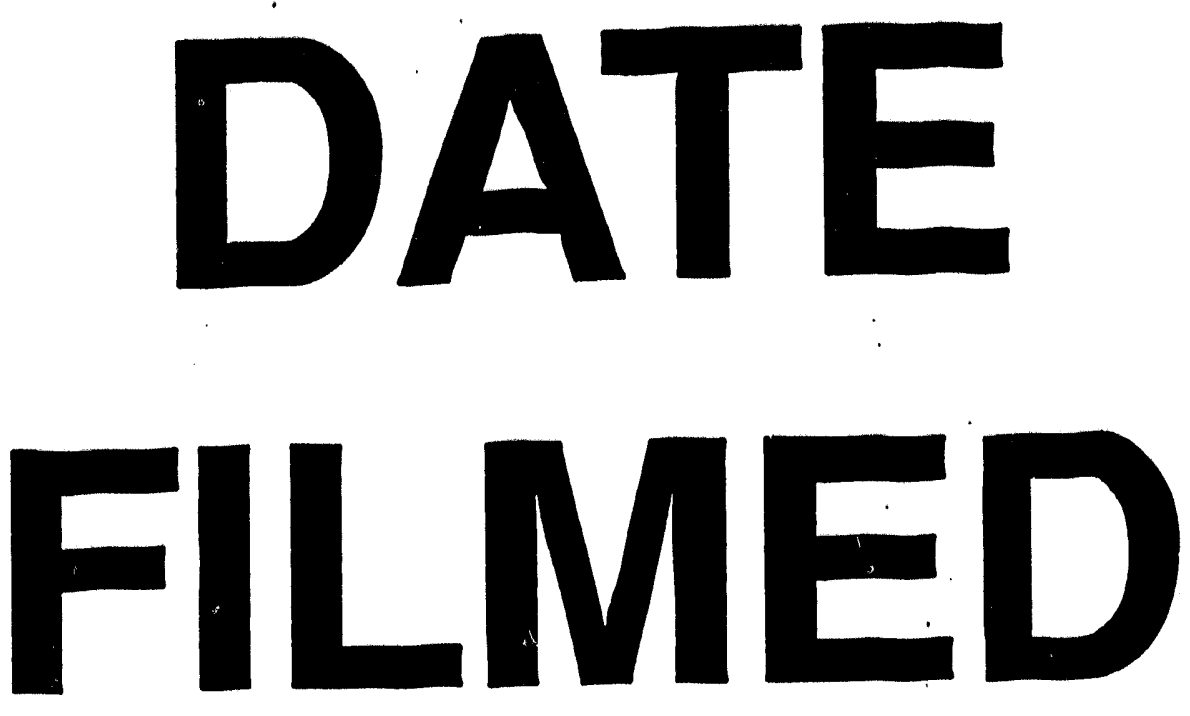

$11 / 17 / 93$
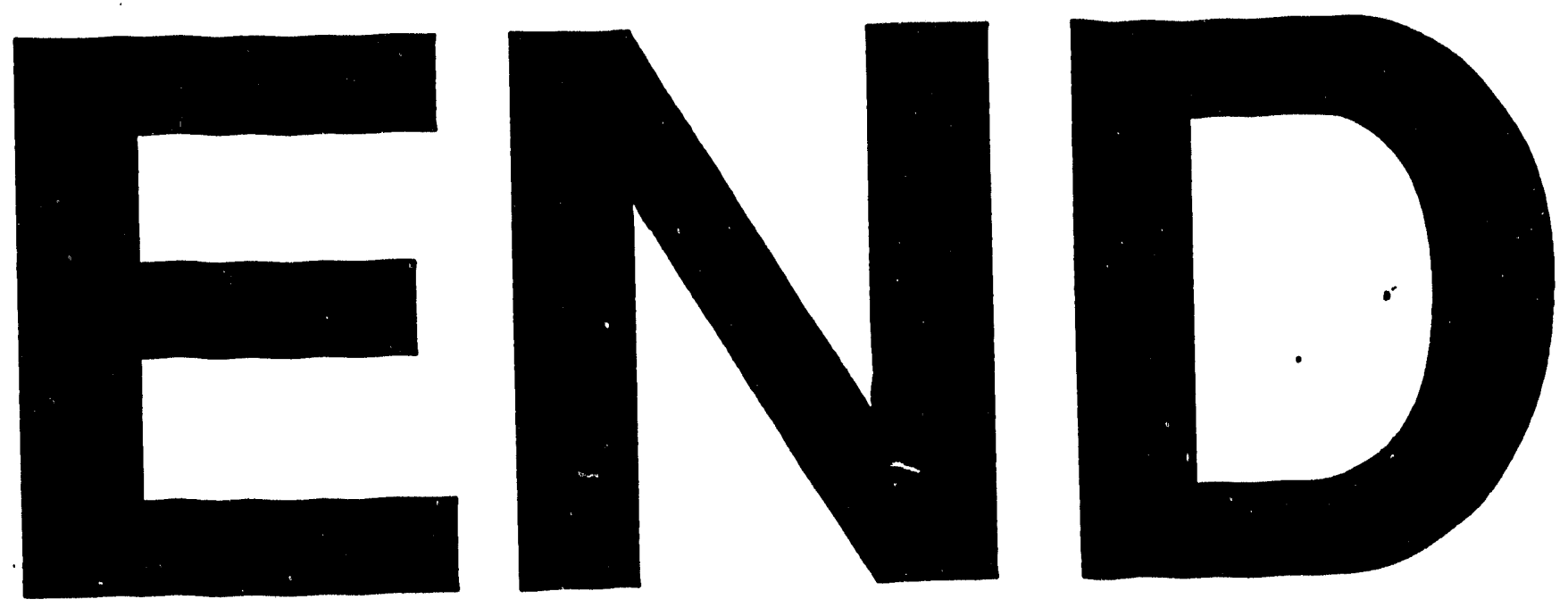
